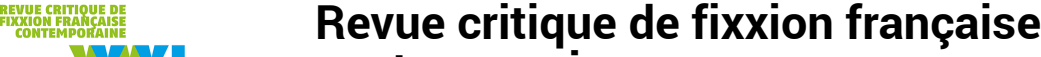 \\ XXI contemporaine

$21 \mid 2020$

Fictions et pouvoirs

\section{Bas les masques}

Forêt contraire d'Hélène Frédérick

\section{Emmanuel Bouju}

\section{(2) OpenEdition \\ Journals}

Édition électronique

URL : https://journals.openedition.org/fixxion/618

DOI : $10.4000 /$ fixxion. 618

ISSN : 2295-9106

Éditeur

Ghent University

Référence électronique

Emmanuel Bouju, "Bas les masques », Revue critique de fixxion française contemporaine [En ligne], 21 । 2020, mis en ligne le 15 décembre 2020, consulté le 17 février 2022. URL : http://

journals.openedition.org/fixxion/618; DOI : https://doi.org/10.4000/fixxion.618

Ce document a été généré automatiquement le 17 février 2022.

\section{(c) $(1) \&$}

Les contenus de la Revue critique de fixxion française contemporaine sont mis à disposition selon les termes de la licence Creative Commons Attribution - Pas d'Utilisation Commerciale - Pas de Modification 4.0 International. 


\title{
Bas les masques
}

\author{
Forêt contraire d'Hélène Frédérick
}

\author{
Emmanuel Bouju
}

Impatient, lonely and shivering,
waiting to build her glorious fires.
A Silver Mt. Zion

1 Parfois, on va dans une forêt pour aller dans une forêt - pour se "déconfiner" comme on dit maintenant - avant de revenir à la ville, au siècle. Parfois, on y va comme si on inversait le monde et récusait, ensauvagé e, la civilité antérieure et la contrainte de ses pouvoirs. Un peu comme Timon d'Athènes fuyant la persécution de la société et le poids de la dette - effets de sa générosité démesurée et déclencheurs de sa folie misanthrope - en se réfugiant dans ce qu'Hélène Frédérick appelle une "forêt contraire" : un lieu inverse, qui contrarie, qui va à l'encontre.

“Quel est l'état du monde si l'accueil / Que l'on me fait est tel, des cris stridents / De créanciers, des traites non payées, des dettes retenues depuis longtemps ?" ${ }^{1}$. Celle qui n'a pas de nom, lorsqu'elle se présente aux premières lignes du roman, a fui son identité antérieure et le poids de ses dettes. Comme Timon, elle "doit pour chaque mot" ${ }^{2}$, et cherche, aux racines du ciel, à récuser la tyrannie de l'or : aussi a-t-elle rejoint la forêt de son enfance, la forêt d'Inverness (au Québec), pour y trouver un temps d'avant l'histoire, un lieu sans pouvoir, un vide où recommencer. Ce faisant, la narratrice cesse d'être "une colonne de chiffres qui se termine en négatif" ${ }^{3}$ : ce à quoi elle avait été réduite dans la civilité de son exil parisien, en tant que "détentrice" - le vocabulaire de l'économie est cruel - d'une dette étudiante, dont l'autrice elle-même a fait la douloureuse expérience pendant de nombreuses années, avant de pouvoir la considérer, enfin, comme une "broutille", une illusion de "pacotille"4

3 Mais ce n'est pas, contrairement à la pièce de Shakespeare ${ }^{5}$, le récit d'une misanthropie fatale et d'une munificence (bounty) dévoyée : c'est le récit d'une impasse commune, couramment éprouvée, pour qui se voit privée, dès sa jeunesse, des moyens d'exister. Parfois, on va à l'université pour devenir étudiant - pour étudier, tout simplement. Pour choisir ce que l'on veut être. Et l'on ne se rend pas compte qu'on se lie étroitement, par la grâce d'un prêt miraculeux, à un devenir que l'on n'a pas choisi. 
4 La logique de la dette exerce un pouvoir qui grève durablement le temps vécu. Et parvenue au mitan de sa vie, on se retrouve dans une forêt obscure sans être guidée pour autant par Virgile - car l'allégorie du péché qu'est devenue la dette dans notre économie néolibérale ne souffre pas de salut.

5 La narratrice entreprend donc de rompre ce lien d'endettement en retournant dans sa forêt d'origine - une forêt ambivalente ${ }^{6}$ où faune et flore définissent les moyens d'exister - comme dans une épiphanie négative typiquement shakespearienne : "À l'échelle de la forêt, ou sous une voûte étoilée, l'humain est bien forcé d'admettre qu'il n'est qu'un insecte" . Fuyant sa demeure à crédit, elle trouve une nouvelle identité d'emprunt - "Sophie" - par l'arbitraire d'un échange social minimal nécessitant de recouvrer un nom - la rencontre (qui s'avérera décisive) avec André. Je narrant, elle passé et nom d'emprunt configurent sa nouvelle demeure, "réinventée" pour l'occasion.

6 Aussi la cabane familiale, ouverte à tous les vents comme la conscience chez Levinas8, devient-elle "une géographie de [son] état présent". Une géographie de "plans sauvages $»^{10}$, où les titres des morceaux musicaux qu'elle affectionne sonnent comme des fragments arrachés aux feux follets de la Tempête: "Born Into Trouble As The Sparks Fly Upward"11. Mais la géographie du présent est aussi réhabilitée par André, le trop bon samaritain du voisinage : lorsqu'il s'offre à retaper la maison et l'aménager pour qu'elle y vive, s'agit-il d'une nouvelle économie du "don" ? Ou d'une nouvelle dette, contractée dans l'implicite d'une relation, de nouveau, inégalitaire?

On se rappelle que Derrida, dans Donner le temps $1^{12}$, avait dénié à la théorie du don/ contre-don de Mauss la capacité à sortir du régime de la dette, et avancé l'impossibilité du don en-dehors de l'expérience d'un présent pur, d'un temps véritablement horspouvoir, d'une pure inconditionnalité. On retrouve ici l'espoir de cette expérience, finir de "squatter les vestiges du passé" et "habiter le présent" ${ }^{13}$ : "André ne semble pas atteint par la question du temps, comme s'il n'en faisait pas pleinement partie, comme s'il évoluait dans la marge, la périphérie de l'Histoire. En lui, tous les temps se conjuguent au présent ; matin, soir, jour et nuit se confondent"14.

Ici, dans cette forêt dés-obscurcie, ce qui se joue, c'est une utopie, l'utopie du temps arrêté et des dettes liquidées.

Utopie impossible, car à dire vrai, le présent de "Sophie" est plus que jamais hanté par un autre passé, le passé d'une autre dette, ce temps montréalais de l'occasion ratée et de la disparition, un temps à la fois advenu et non advenu. Son présent est hanté par la douleur fantôme de ce qui n'a pas eu lieu : le salut de Lukas Bauer, un écrivain que la narratrice n'a pas pu sauver du suicide - et double fictif de Lothar Baier, l'écrivain et essayiste allemand, connue de l'autrice et mort également par suicide le 11 juillet 2004 à Montréal ${ }^{15}$.

Cette utopie fait écho aux Liens, le manuscrit de Lukas Bauer, seul rescapé du monde d'avant, qu'a emporté avec elle la narratrice. C'est l'utopie d'un temps hors de ses gonds, qui ressemble dangereusement à la tentation de la disparition recommencée. Car comme le remarque la narratrice-lectrice des Liens, le livre porte "en exergue, une phrase de Thomas Bernhard : 'Un jour, en un unique instant nous enfoncerons la frontière ultime mais le moment n'est pas encore là. Nous connaissons la méthode, mais le moment, nous ne le connaissons pas"'16.

11 Est-ce là la méthode pour sortir de la forêt ? Ou bien plutôt pour y disparaitre? 
C'est là le moment d'une tentation, celle d'une réplique radicalement solipsiste à la domination des pouvoirs : “D’un regard extérieur autant qu'intérieur, du général basculant par moments dans l'intime, Lukas avait étudié les conséquences d'une domination économique, mais en abordant de biais la lancinante volonté d'en finir avec soi"17. C'est là l'envers à la fois personnel et bibliographique du roman : son écho secret aux œuvres de Lothar Baier - comme Keine Zeit. 18 Versuche über die Beschleunigung ${ }^{18}$, et surtout Die verleugnete Utopie. Zeitkritische Texte ${ }^{19}$.

Soit : l'utopie reniée, rompue, avortée. Telle une forêt contraire.

Car ce que raconte aussi le roman d'Hélène Frédérick, c'est la nécessité de porter l'utopie jusqu'à son point de rupture. Jusqu'au point de rupture des Liens dont elle a hérité et dont il faut se défaire, tout en les faisant jouer entre ses doigts ${ }^{20}$. Tel est le principe énonciatif et représentationnel du roman : rejouer et dénouer, dans le présent artificiel de cette forêt contraire, un passé contrarié. C'est le pari d'une puissance de la représentation contre l'atteinte du pouvoir. Cette puissance du signe qui revient aujourd'hui comme retournement du stigmate.

Pour lui donner forme sensible, j'ai avancé ailleurs l'idée du modèle de la "boîte miroir"21 qui permet de remobiliser les articulations du passé, et ici de faire jouer le désir sensible en lieu et place de la douleur fantôme. C'est ce que propose André : “On va jouer avec le temps et les souvenirs. Je l'écoutais sans rien dire, sidérée par ce discours inattendu. [...] On ne fera pas parler les morts, on fera parler des personnages ; j'inventerai, avec vous, un homme hors du réel. Pas de profanation, puisqu'on entre dans la fiction, dans le jeu, on est bien d'accord" ${ }^{22}$.

Pièce dans la pièce, boîte-miroir dans la cabane de la conscience, la cérémonie des masques libère la persona du passé, et la rend presque "translucide" ${ }^{23}$, comme si le masque formait une seconde peau. Jusqu'à faire apparaître le visage sur le masque, comme disait Leonardo Sciascia. Jusqu'au risque du suicide recommencé.

7 L'utopie est rompue, par la menace contenue dans cet envers du monde - encore marqué par le pouvoir de l'endettement et la fausse solution du suicide. Car retourner la parole comme un gant, c'est un geste dont la puissance ne suffit pas toujours contre l'exercice du pouvoir: "André était-il en train de me faire rembourser ma dette et de s'approprier indûment mon passé ? [...] Ces paroles ont beau être faites de mots inoffensifs, on dirait qu'elles portent en elles, en négatif, tout l'enfer qu'elles vous feront vivre. On les retourne comme on retourne un gant et l'avenir est inscrit là, juste au bord de l'ourlet. Pareil au nom que votre mère y aurait cousu, il vous saute aux yeux, en noir sur blanc"24.

8 Comme l'écrivait Barthes dans "Le refus d'hériter" : "abolir le récit, c'est dépasser le fantasme : il faut concevoir l'écrivain (ou le lecteur : c'est la même chose) comme un homme perdu dans une galerie de miroirs : là où son image manque, là est la sortie, là est le monde" 25 .

9 Quand la cabane de la conscience est redevenue une galerie des glaces, un jeu de miroirs en forme d'impasse, il faut savoir coûte que coûte en sortir. Il faut, in fine, par refus radical d'hériter et liquidation des dettes, contrarier la forêt originelle, et dire : bas les masques.

Ainsi Hélène Frédérick achève-t-elle sa Forêt contraire en un trait de plume et de feu, dans lequel résonne encore, à qui sait l'entendre - Built Then Burnt [Hurrah! Hurrah!] - la musique des Silver Mt. Zion. 


\section{NOTES DE FIN}

1. "How goes the world, that I am thus encountered with clamorous demands of long-since-due debts [...]?" (William Shakespeare, La vie de Timon d'Athènes, trad. André Marcowicz, Les Solitaires intempestifs, 2005, p. 65)

2. "He owes / For every word" (ibid., p. 57).

3. Hélène Frédérick, Forêt contraire, Paris, Gallimard/Verticales, 2014, p. 26.

4. Ibid, p. 26.

5. Pièce dont la condamnation de la chrématistique a pu inspirer jusqu'à Marx, et dont le vœu d'une "peste planétaire" résonne à nos oreilles comme une malédiction archaïque.

6. Inverness est le nom du château de Macbeth.

7. Hélène Frédérick, op. cit., p. 60.

8. Emmanuel Levinas, "Sans nom", Noms propres, St-Clément de Rivière, Fata Morgana, 1976, p. 181.

9. Hélène Frédérick, op. cit., p. 98.

10. Titre du recueil de poésie paru en 2016 aux éditions montréalaises L'Oie de Cravan.

11. Hélène Frédérick, op. cit., p. 99. C'est le titre d'un album de Thee Silver Mt. Zion Memorial Orchestra (ou A Silver Mt. Zion), un groupe de postrock montréalais.

12. Jacques Derrida, Donner le temps. 1. La fausse monnaie, Paris, Galilée, 1991.

13. Hélène Frédérick, op. cit., p. 91.

14. Ibid., p. 71.

15. Voir l'article de Robert Dion, qui fait du roman un reliquaire de l'œuvre de Lothar Baier : "Roman à clés et écriture du réel : La constellation du Lynx de Louis Hamelin et Forêt contraire d'Hélène Frédérick", Eurostudia, vol. 13 n 1-2, 2018-2019, p. 379

16. Hélène Frédérick, op. cit., p. 46.

17. Ibid., p. 61.

18. Lothar Baier, Keine Zeit. 18 Versuche über die Beschleunigung, Munich, Antje Kunstmann, 2000. Traduit en français sous le titre Pas le temps! Traité sur l'accélération (2002).

19. Lothar Baier, Die verleugnete Utopie. Zeitkritische Texte, Berlin, Aufbau, 1993.

20. "un décor de lambeaux de liens rompus avec les miens, je veux dire ceux de l'origine, le contraire de nœuds coulants qui étoufferaient" (Hélène Frédérick, op. cit., p. 98).

21. Emmanuel Bouju, "A Nest in the Air: Phantom Pain and Contemporary Narrative", dans Lia Brozgal et Sara Kippur (eds), Being Contemporary: (Un)timely Essays in French Culture, Liverpool, Liverpool University Press, 2015, p. 349-361. Voir aussi Épimodernes. Nouvelles "leçons américaines" sur l'actualité du roman, Québec, Codicille éditeur, 2020.

22. Hélène Frédérick, op. cit., p. 129.

23. Ibid., p. 150. C'est pousser à ses limites la différence originaire entre masque et visage par laquelle le concept de "personne" a pu fonder sa prédominance théologique et juridique : voir la reprise des thèses de Simone Weil chez Roberto Esposito, "Pour une philosophie de l'impersonnel”, dans Communauté, immunité, biopolitique, Sesto S. Giovanni, Editions Mimésis, 2019, p. 242.

24. Hélène Frédérick, op. cit., p. 148-149. Retourner la parole comme un gant, ou comme Benjamin enfant sa chaussette, pour découvrir l'identité entre forme et contenu (Enfance à Berlin). Que devient le prénom de Benjamin, aurait dit Derrida, une fois la chaussette d'enfant retournée ?

25. Roland Barthes, "Le refus d'hériter", dans Euvres complètes, t. 5, Paris, Éditions du Seuil, 2002 [1968], p. 603. 
AUTEURS

EMMANUEL BOUJU

Université de la Sorbonne Nouvelle 